\title{
Innovación en Chile como aporte a la democratización de los servicios: análisis del caso de Gendarmería de Chile
} Innovation in Chile as a contribution to the democratization of services: analysis of the
Chilean Gendarmerie case

\author{
Gabriela Guevara y Julio Hasbún
}

Universidad de Chile

\section{América Latina y Chile, vías de innovación}

Lo que hoy denominamos innovación fue entendida en la posguerra como parte del proceso de industrialización en Latinoamérica, en que la inversión en investigación y desarrollo permitiría la obtención de procesos y productos que a su vez generarían grados crecientes de competitividad en dichos países ${ }^{1}$. Siendo esta perspectiva la piedra angular del proceso de innovación, poniendo al centro la formación de capital humano y tecnología, en este ensayo sostenemos que dicho enfoque es necesario, más no suficiente para responder a los desafíos de la democracia en la América Latina del siglo XXI.

Dada la heterogeneidad asincrónica de estos procesos de modernización en los países de nuestro continente, nos situaremos específicamente en el caso de Chile, tomando como caso de estudio el proceso de instalación de una plataforma de innovación en Gendarmería.

En la posguerra (década de los cincuenta y setenta del siglo pasado), la política económica de sustitución de importaciones invirtió en esta visión de desarrollo científico y tecnológico, basada en una noción reducida del desarrollo que lo entendía eminentemente como crecimiento económico (CEPAL, 2000), esfuerzo que consistió más en transferencia tecnológica desde los países industrializados, que en creación de conocimientos locales, y que se vio truncado por la crisis económica y la instauración de dictaduras militares. Durante los setentas y ochentas además, estos esfuerzos desconectaron a nuestros países de los cambios sociales y culturales que estaban gestándose en los centros económicos, donde la ciudadanía presionaba por un mayor espacio entre el Estado y el mercado.

El retorno a la democracia, en términos generales, se desarrolla en contextos de ajuste estructural

\footnotetext{
${ }^{1}$ Estrictamente, la competitividad en el largo plazo es una característica de las regiones, no de los países.

*Dirección de correspondencia [Correspondence

address]: Julio Hasbún, Universidad de Chile

E-mail: jhasbun@gmail.com
}

de los Estados latinoamericanos, plasmados en el Consenso de Washington, en que el abandono de Keynes marca el retorno de concepciones más liberales expresadas como: disciplina fiscal, privatización de la empresa fiscal, liberalización comercial y financiera, cambios en estructura del gasto, entre otras (Ramírez Brouchoud, 2009), siendo Chile un ejemplo paradigmático.

Esta economía neoliberal, en que el repliegue del Estado da espacio a la entrada de nuevos actores económicos en los nuevos mercados producto de la privatización del fisco, haría caer la pobreza mediante el ?chorreo? hacia las capas más carenciadas de la sociedad. Como resultado, dichas políticas permitieron el crecimiento económico gracias a la demanda de materias primas de los países emergentes, pero también ahondaron las desigualdades estructurales de la economía latinoamericana, generando malestar, desconfianza e inseguridad, producto de la pérdida del tejido social que provocaron los gobiernos autoritarios al abandonar a las clases medias a su suerte ${ }^{2}$, pasando desde un modelo tradicional más cooperativo a uno basado en la competencia, dejando a ganadores y perdedores segmentados social y espacialmente, por ejemplo en Chile, gracias a erradicaciones de poblaciones de menores recursos hacia los márgenes de las ciudades. Esta polarización y desintegración social desnuda la incapacidad del Estado para responder con mecanismos democráticos (Lechner, 1992) por lo que reprimirá la reivindicación social, reduciendo la capacidad de la ciudadanía para demandar y protestar, desmembrando a los sindicatos y otras formas de organización social ciudadana, generando mayores niveles de asimetría.

El diagnóstico del Banco Mundial para América Latina es bastante claro para 1997: estos procesos generaron sociedades polarizadas con tendencia a la anomia, poniendo en riesgo el orden social y las condiciones de gobernabilidad (Ramírez Brouchoud, 2009), gracias a lo cual se propondrán una

\footnotetext{
${ }^{2} \mathrm{El}$ retroceso del Estado como actor empresarial a un mero rol ?subsidiario? lo hará contar con menos recursos, por lo que en adelante focalizará sus recursos hacia los grupos más vulnerables, como una forma de contener la creciente protesta social de aquellos grupos "perdedores", entre ellos una clase media pauperizada que siempre está a un paso de descender en la escala social.
} 
nueva serie de reformas de ?segunda generación? al Estado, conocidas como las ?cuatro E?: eficacia, eficiencia, equidad y entorno propicio para el mercado, basadas en el New Public Management propugnado por OCDE, aplicado especialmente en Australia, Inglaterra y Nueva Zelanda. En esta visión el Estado comienza a aplicar recetas provenientes del ámbito de la gestión privada con el fin de superar la inercia burocrática, buscando su modernización (Ramírez-Alujas, 2011). Este management en Latinoamérica tendrá magros resultados, fortaleciendo actitudes aislacionistas entre los organismos del Estado (ahora compiten entre sí), desincentivando la coordinación, lo cual elevará los costos de transacción de los ciudadanos y la sociedad en general. Esta ?imitación? de prácticas de gestión privada en el Estado debe dar paso a una ?innovación? que permita el desarrollo de capacidades macroorganizativas nuevas, como la creación de redes interinstitucionales, favoreciendo la coordinación entre las organizaciones, y la articulación de la acción de los organismos del Estado y su vinculación con otros actores (Metcalfe, 1996).

En este contexto, aun cuando la política pública de ciencia y tecnología puede facilitar el desarrollo de una cultura democrática para nuestros países, al incorporar como objetivos la cohesión social y ciudadanía (García Guzmán, 2011), especialmente mediante la disminución de la pobreza, se hace necesario que el Estado responda a las nuevas condiciones sociales, económicas y culturales, caracterizadas por la emergencia de nuevos ?problemas malditos? (marginalidad juvenil, generación NINI, cambio climático, cambios demográficos, entre otros), y una ciudadanía con mayores niveles educativos que, consciente de sus derechos, los demanda dejando atrás la mirada de ?beneficios?, exigiendo grados mayores de transparencia y rendición de cuentas ${ }^{3}$. Para ello, el Estado debe buscar el desarrollo de eficiencias y mejorar la calidad de los servicios que presta a la ciudadanía. Es en este contexto que la innovación juega un rol fundamental en el sector público.

Para la OECD (2009) la innovación es "la aplicación de una nueva o significativa mejora en un producto (bien y/o servicio) o proceso". Rápidamente, ha pasado de ser una moda a un imperativo de supervivencia para la competitividad de las empresas, países y finalmente, los Estados y sus instituciones. En este escenario, la innovación en el sector público contribuye de manera significativa a la generación de valor público, a la apertura y democratización de

\footnotetext{
${ }^{3}$ La inclusión de Chile en OCDE ha sido fundamental para el avance en estas materias. Por ejemplo, la creación del Consejo Para la Transparencia (2009) fue parte de los compromisos adquiridos por el país ante dicha instancia multilateral.
}

los servicios, toda vez que supone en la génesis de sus procesos elementos de análisis y desarrollo fundados en la consideración de los ciudadanos como actores clave. "La innovación es fundamental para permitir que las sociedades construyan los nuevos modelos de gobierno y de gobernabilidad que necesitamos para abordar los grandes retos del siglo XXI. Queda claro que los gobiernos que sean capaces de enfrentar estos retos no lo harán con métodos lentos y evolutivos, sino mediante niveles sin precedentes de innovación y liderazgo" (Gowher, 2009).

\section{Innovación de servicios públi- cos: el pensamiento de diseño (design thinking en Gendar- mería de Chile)}

Gendarmería de Chile es el servicio público con el mayor número de funcionarios del país (casi diez y seis mil), que enfrenta problemas importantes dado el crecimiento de la población penal de las últimas décadas, con accidentes como el incendio de la Cárcel de San Miguel (2010) que cobró la vida de ochenta y una personas, que ponen en el tapete sus prácticas de reclusión, seguridad, condiciones de higiene, y calidad de vida tanto de sus internos como la de sus funcionarios.

La complejidad de la misión que este servicio realiza (reclusión, atención y reinserción de sus usuarios) abre espacios importantes para mejorar e innovar en las condiciones de sus prestaciones y productos.

En este contexto, por primera vez en la historia de la Corporación de Fomento de la Producción (CORFO) el año 2012 abre un concurso para apoyar la generación de procesos permanentes de innovación en el sector público, apoyando a diez servicios a lo largo del país, obteniendo así Gendarmería de Chile recursos para implementar su plataforma de innovación, y capacitar a una masa crítica de funcionarios con el objetivo de dar continuidad a estos esfuerzos mediante la constitución de una cultura de la innovación dentro del servicio. En una alianza de Gendarmería con Consultora Holos S.A. se definió la aplicación de una metodología de Pensamiento de Diseño para capacitar a sus funcionarios.

La metodología de Pensamiento de Diseño consiste en una aproximación humano-céntrica basada en el diseño con el objetivo de ayudar a las organizaciones del sector público y privado a innovar y crecer. Posee cinco fases que completan un ci- 
clo iterativo: empatizar, definir, idear, prototipar y evaluar. Como sitúa la experiencia de los usuarios en el centro es especialmente apta para el diseño de servicios públicos. Además, el diseño de servicios se basó en cinco principios (Stickdorn y Schneider, 2012): (i) centrado en el usuario: los servicios deben experimentarse a través de los ojos de los usuarios; (ii) co-creativo: todos los involucrados deben ser incluidos en el diseño del servicio; (iii) secuencial: el servicio debe ser visualizado como una secuencia de acciones interrelacionadas; (iv) evidenciar: los servicios intangibles deben ser visualizados en términos de artefactos concretos; y (v) holístico: el entorno completo de los servicios debe ser considerado.

Para su aplicación en Gendarmería la metodología fue adaptada inicialmente para involucrar en la definición de focos de innovación a las distintas unidades y posiciones en la jerarquía de la organización de acuerdo a un diagnóstico realizado con diversas técnicas (entrevistas, grupos focales, encuestas en línea, entrevistas en profundidad) que entregó alrededor de sesenta y cinco focos que fueron presentados al Director Nacional de la organización, con el objeto de seleccionar los doce finales, en torno a los que se constituyeron equipos heterogéneos de entre cinco y diez personas, cada uno con un coordinador de equipo y responsables institucionales ${ }^{4}$.

A partir de allí comenzó una segunda fase de talleres, asociada al ciclo del Pensamiento de Diseño, basada en el ?aprender haciendo?. En primer lugar se llevó a cabo un taller de observación y comprensión empática, con el objetivo de comprender las necesidades de los usuarios a través de herramientas como la entrevista in situ, mapas de empatía, entre otras, para finalmente definir en forma precisa cuál es la oportunidad-necesidad que se buscará abordar.

En segundo lugar, se realizó un taller de pensamiento creativo con el fin de desarrollar la mayor cantidad de ideas y conexiones novedosas en relación a la oportunidad-necesidad abordada, para lo que se aplicaron herramientas como lluvia de ideas y pensamiento lateral, entre otras. Al tener muchas ideas, mediante criterios como impacto, factibilidad, costos, es posible seleccionar la más adecuada para la institución, que puede ser una tanto una mejora como una innovación.

En tercer lugar los funcionarios realizaron un taller de prototipado, con el fin de entender la importancia de probar y fallar en forma económica

\footnotetext{
${ }^{4}$ Siguiendo a Koch y Hauknes (2006) que sugiere que las personas innovan (entre otras razones) motivados por la búsqueda de soluciones a probemas puntuales, específicos y acotados, identificados por ellas mismas.
}

mediante prototipos (simulaciones) de la mejora o innovación seleccionada, haciendo que los usuarios retroalimenten el proceso, para ir mejorando el prototipo.

En cuarto lugar, se llevó a cabo un taller de gestión de proyectos a fin de entregar una serie de herramientas para desarrollar un primer plan de implantación de los prototipos de mejoras o innovaciones seleccionados, de manera de darles sostenibilidad.

Cada taller contó con especialistas, y se realizaron uno cada mes, dando tiempo a los equipos de ir aplicando las herramientas entregadas en cada taller que fuesen pertinentes a sus desafíos.

El nivel de avance de los equipos fue diverso, y en nuestra experiencia fue fundamental el liderazgo y compromiso ejercido por los coordinadores, puesto que todos estaban con sus cargas laborales a tope, y debían mostrar capacidad de emprender para encontrar espacio, y a la vez cohesionar el trabajo de sus equipos.

Paralelamente al ciclo del Pensamiento de Diseño se llevaron a cabo las siguientes actividades: (1) constitución de unidad de innovación en Gendarmería; (2) implementación de una plataforma de comunicación virtual para la innovación; (3) concurso interno para el diseño del logotipo de la unidad de innovación; (4) diseño del proceso de innovación institucional.

\section{Aprendizajes del proceso de in- novación en Gendarmería}

La potencialidad de la metodología aplicada radicó en los siguientes elementos:

1. Obligó a los funcionarios a empatizar y experienciar los impactos del servicio en las vidas de sus usuarios, repensando sus roles y generando motivación interna para el desarrollo de sus tareas. Los usuarios pueden ser internos y externos, es decir, incluye a todos los funcionarios del servicio.

2. Reactivó en los funcionarios la capacidad para poner en marcha su creatividad para encontrar soluciones que mejoren su labor y las vidas de sus usuarios.

3. Al constituirse equipos interdisciplinarios para dar respuesta a los desafíos, facilitó el encuentro entre distintas personas y funciones, lo que genera pertenencia e identidad en la organización. 
4. En un contexto en el que los problemas que enfrenta la sociedad, y por tanto, la institución, se van complejizando (transición demográfica, marginalidad juvenil, cambio climático, entre otros) y los recursos son siempre escasos, la innovación apuntó a hacer más con menos, generando eficiencias basadas en un concepto ético de servicio público.

5. Ante una ciudadanía cada día más exigente y empoderada, es fundamental mejorar los servicios para entregar respuestas satisfactorias a las demandas ciudadanas, razón por la que innovar pensando en las personas se constituye en una oportunidad para democratizar el servicio público.

Las dificultades observadas para aplicar la metodología apuntaron en dos direcciones:

1. La sobrecarga laboral de los funcionarios, que impedía muchas veces la participación sistemática en los talleres y tareas de cada equipo de innovación. En este sentido el involucramiento de los directivos de la organización es central para alinear a los mandos medios para que permitan asignar tiempos a los funcionarios para asistir a los talleres y reuniones de equipo (Waissbluth 2008).

2. La excesiva burocracia en los procesos, ya que cada acción que debía ser visada por los directivos del servicio, tomaba semanas para ser aprobada, entorpeciendo la continuidad de la implementación de las innovaciones.

Finalmente, es relevante mencionar que los funcionarios públicos tienen una necesidad de conversar y reflexionar sobre su trabajo, ya que se ven involucrados en las vidas de sus usuarios, y en el espacio de sus tareas no tienen el tiempo ni el espacio para desarrollar meta cogniciones acerca de sus roles. El capital humano es el activo central de los servicios públicos en las economías modernas, en este sentido la experiencia narrada en Gendarmería de Chile demostró que los prejuicios que se tienen sobre el funcionarios público están asociados a la falta de sentido de su tarea, pues cuando ésta se vuelve impersonal y mecánica, se pierde la cualidad humana que es la base del proceso de co-creación en que se sustenta el servicio.

\section{Referencias}

CEPAL (2000). Equidad, desarrollo y ciudadanía. CEPAL-Alfaomega, Santiago de Chile.
García Guzmán, M. (2011). Políticas de innovación científica y tecnológica en América Latina. Encrucijada. Revista Electrónica del Centro de Estudios en Administración Pública.

Gowher, R. (2009). Ash Center for Democratic Governance and Innovation. Harvard Kennedy School of Government - KSG.

Koch, P. y Hauknes, J. (2006). On innovation in the public sector - today and beyond. PUBLIN Report No.D20, NIFU STEP (second revised edition), Oslo.

Lechner, N. (1992). El debate sobre Estado y Mercado. Nueva Sociedad, (121):80-89.

Ramírez-Alujas, A. V. (2011). Sobre la aplicación y desarrollo del concepto de innovación en el sector público: Estado del arte, alcances y perspectivas. Revista Circunstancia, IX(26).

Ramírez Brouchoud, M. F. (2009). Las reformas del Estado y la administración pública en América Latina y los intentos de aplicación del New Public Management. Estudios Políticos, (34):115-141.

Stickdorn, M. y Schneider, J. (2012). his is service design thinking: basics, tools, cases. Wiley, New Yersey. 